\title{
Radio-frequency optomechanical characterization of a silicon nitride drum
}

\author{
A. N. Pearson ${ }^{1}$, K. E. Khosla ${ }^{2,3}$, M. Mergenthaler ${ }^{1}$, G. A. D. Briggs ${ }^{1}$, E. A. Laird ${ }^{4}$ \& N. Ares ${ }^{1 *}$ \\ On-chip actuation and readout of mechanical motion is key to characterize mechanical resonators and \\ exploit them for new applications. We capacitively couple a silicon nitride membrane to an off resonant \\ radio-frequency cavity formed by a lumped element circuit. Despite a low cavity quality factor $\left(Q_{E} \approx 7.4\right)$ \\ and off resonant, room temperature operation, we are able to parametrize several mechanical modes \\ and estimate their optomechanical coupling strengths. This enables real-time measurements of the \\ membrane's driven motion and fast characterization without requiring a superconducting cavity, \\ thereby eliminating the need for cryogenic cooling. Finally, we observe optomechanically induced \\ transparency and absorption, crucial for a number of applications including sensitive metrology, ground \\ state cooling of mechanical motion and slowing of light.
}

Cavity optomechanics boasts a number of tools for investigating the interaction between radiation and mechanical motion and enables the characterization and development of highly sensitive devices ${ }^{1}$. Silicon nitride membranes have been fabricated to exhibit very high tensile stress, resulting in high quality factors, and have been used for a number of applications including measurement of radiation pressure shot noise ${ }^{2}$, optical squeezing ${ }^{3}$, bidirectional conversion between microwave and optical light ${ }^{4}$, optical detection of radio waves ${ }^{5,6}$, microkelvin cooling ${ }^{7}$ and cooling to the quantum ground state of motion ${ }^{8-10}$.

Radio-frequency (rf) cavities allow for sensitive mechanical readout on-chip ${ }^{5,11-14}$. We characterize a silicon nitride membrane at room temperature making use of an off-resonant $\mathrm{rf}_{\text {cavity }}{ }^{13,14}$. In our approach, the use of lumped elements greatly simplifies the detection circuit in terms of fabrication and allows the integration on chip with the mechanical oscillator. Our circuit has a lower operation frequency than microwave cavities ${ }^{11,14}$, and allows for a larger readout bandwidth than previous works ${ }^{13}$. Also, our cavity allow us to inject noise, effectively increasing the mechanical mode temperature. We are able to detect several modes and extract the quality factor and cavity coupling strength for each of them. When the membrane is driven, we are able to resolve the membrane's motion in real time. We achieve a sensitivity of $0.4 \mathrm{pm} / \sqrt{\mathrm{Hz}}$. A sensitivity of $4.4 \mathrm{pm} / \sqrt{\mathrm{Hz}}$ was reported in ref. ${ }^{14}$, although it must be noted that these sensitivities cannot be easily compared, due to the much smaller size of their mechanical resonator. We observe optomechanically induced transparency (OMIT) and optomechanically induced absorption (OMIA) on-chip and deep in the unresolved sideband regime, allowing for the characterisation of the membrane's motion under radiation pressure. OMIT and OMIA are an unambiguous signature of the optomechanical interaction ${ }^{15}$ and they can be used to slow or advance light ${ }^{16}$. OMIT has also been proposed as a means to achieve ground state cooling of mechanical motion in the unresolved sideband regime ${ }^{17,18}$.

\section{Experiment}

Our device consists of a high-stress silicon nitride membrane which is $50 \mathrm{~nm}$ thick; it has an area of $1.5 \mathrm{~mm} \times 1.5 \mathrm{~mm}$ and $90 \%$ of this area is metalized with $20 \mathrm{~nm}$ of Al. We suspend this membrane over two $\mathrm{Cr} / \mathrm{Au}$ electrodes patterned on a silicon chip. A dc voltage $V_{\mathrm{dc}}=15 \mathrm{~V}$ is applied to electrode 1 , with electrode 2 grounded. Measurements were performed at room temperature and at approximately $10^{-6} \mathrm{mbar}$. For optomechanical readout and control, electrode 1 is connected to an effective rf cavity. The cavity is realised using an on-chip inductor $L$ and capacitors $C_{\mathrm{D}}$ and $C_{\mathrm{M}}$ mounted on the sample holder ${ }^{19}$, in addition to the capacitance formed by the membrane $C_{\mathrm{C}}$. The circuit behaves similarly to a simple $\mathrm{LC}$ resonator with total capacitance $C_{\mathrm{T}}=C_{\mathrm{C}}+C_{\mathrm{P}}$ where $C_{\mathrm{P}}$

\footnotetext{
${ }^{1}$ Department of Materials, University of Oxford, Parks Road, Oxford, OX1 3PH, United Kingdom. ${ }^{2}$ Center for Engineered Quantum Systems, The School of Mathematics and Physics, The University of Queensland, St. Lucia, Queensland, 4072, Australia. ${ }^{3}$ OOLS, Blackett Laboratory, Imperial College London, London, SW7 2AZ, United Kingdom. ${ }^{4}$ Department of Physics, Lancaster University, Lancaster, LA1 4YB, United Kingdom. *email: natalia. ares@materials.ox.ac.uk
} 
(a)
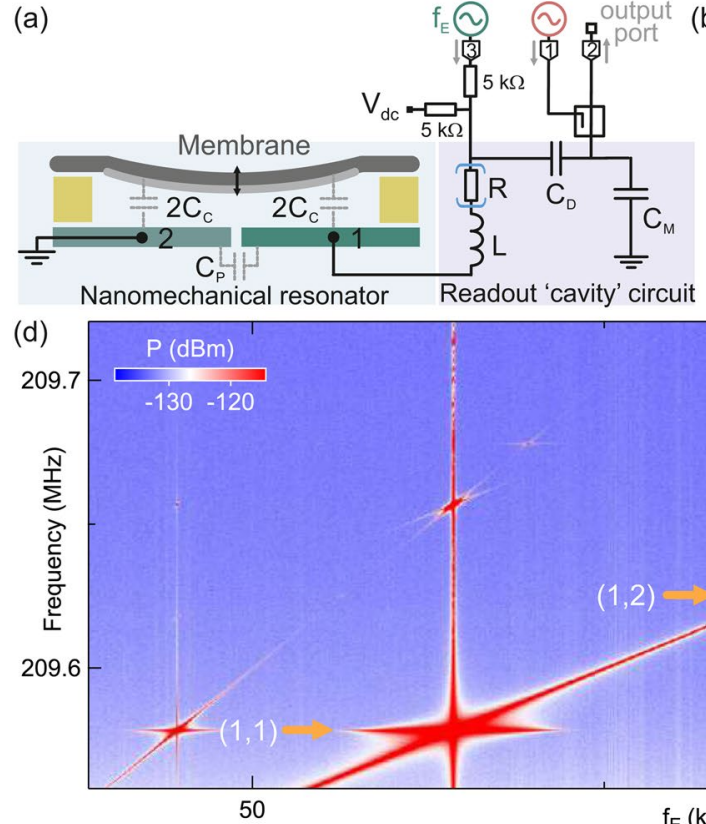

Readout 'cavity' circuit
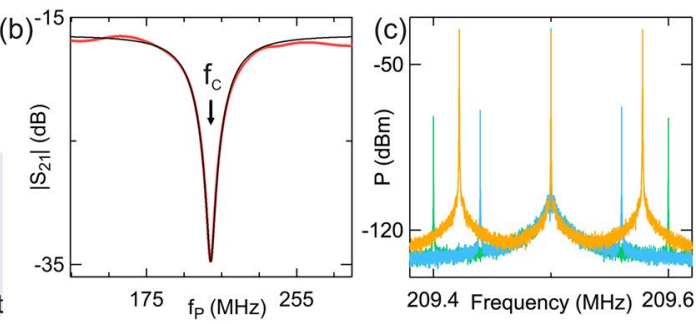

209.4 Frequency (MHz) 209.6

Figure 1. (a) Experimental setup. A metalized silicon nitride membrane is suspended over two metal electrodes. Electrode 2 is grounded and electrode 1 is connected to a radio-frequency tank circuit acting as a readout cavity. The cavity is formed from an inductor $L=223 \mathrm{nH}$ and $10 \mathrm{pF}$ fixed capacitors $C_{\mathrm{D}}$ and $C_{\mathrm{M}}$. Parasitic capacitances contribute to $C_{\mathrm{M}}$. The role of $C_{\mathrm{M}}$ is to improve on the impedance matching between the circuit and the $50 \mathrm{Ohm}$ line. $C_{\mathrm{D}}$ controls the coupling to the cavity, i.e. the number of photons entering the cavity. Because these capacitors are larger than $C_{\mathrm{T}}$, they do not strongly affect the cavity frequency. Parasitic losses in the circuit are parameterized by an effective resistance $R$. To probe the cavity a radio-frequency signal is injected at port 1 , passed via a directional coupler, and after reflection received at port 2 . The signal is measured using a vector network analyser or spectrum analyser. The membrane's motion is excited via port 3. Bias resistors allow a dc voltage $V_{\mathrm{dc}}$ to be added to the membrane drive signal. (b) Reflection as a function of frequency. The black curve is a fit to a circuit model. (c) Power spectrum of the signal at port 2 showing the carrier peak at the cavity resonance frequency, applied via port 1, and sidebands corresponding to a frequency modulated tone applied via port 3 . The light blue and light green curves correspond to the power spectrum for an excitation $f_{\mathrm{E}}$ below $(60 \mathrm{kHz})$ and above $(100 \mathrm{kHz}) f_{0}$ respectively. Sidebands appear due to non-linearities in the circuit. The orange curve corresponds to $f_{\mathrm{E}} \sim f_{0}$. In this case, the sidebands are larger. (d) Higher frequency sidebands in (c) as a function of $f_{\mathrm{E}}$. When $f_{\mathrm{E}}$ is close to a mechanical mode of the membrane, a mechanical sideband appears. The four visible mechanical modes are indicated with arrows. Because higher modes produce fainter features, they are not displayed in this graph. Note that the colour scale displays a range of only $25 \mathrm{dBm}$ in order to reveal the weaker features. Insets: zoom-in of the two highest frequency modes displayed. We observe a splitting of the mechanical feature, indicating nearly degenerate modes.

accounts for the capacitance between the two sides of the antenna and other parasitic capacitances. This circuit acts as a cavity and can be driven by injecting an rf signal to the input (port 1) via a directional coupler in order to induce an optomechanical interaction between the cavity signal and the mechanical motion. In addition port 3 allows injection of an ac signal to directly excite the membrane's motion. The entire setup forms a three-terminal circuit with input ports 1 and 3 and output port 2 (Fig. 1(a)). We used a vector network analyzer to measure scattering parameters and a spectrum analyzer to measure power spectra. Figure 1(b) shows the scattering parameter $\left(\left|\mathrm{S}_{21}\right|\right)$ as a function of cavity probe frequency $f_{\mathrm{p}}$. The cavity resonance is evident in reflection as a minimum in $\left|S_{21}\right|$ with quality factor $Q_{\mathrm{E}} \approx 7.4$.

The dependence of the capacitor formed between the electrodes and the metalized membrane $C_{\mathrm{C}}$ on the mechanical displacement $u$ leads to coupling between the cavity and the mechanical motion. This coupling is given by $\frac{d f_{\mathrm{C}}}{d u} \approx 1 /\left(4 \pi \sqrt{L C_{\mathrm{T}}^{3}}\right) \frac{\partial C_{\mathrm{C}}}{\partial u}$, where $f_{\mathrm{C}}$ is the cavity resonance frequency, $C_{\mathrm{T}}$ the total circuit capacitance and $u$ the membrane's displacement from its equilibrium position. The coupling causes phase shifts of the cavity reflection, allowing us to monitor the membrane's motion in the unresolved sideband limit. The single-photon coupling strength, which measures the interaction between a single photon and a single phonon, is therefore,

$$
\frac{g_{0}}{2 \pi} \approx \frac{1}{4 \pi \sqrt{L C_{\mathrm{T}}^{3}}} \frac{\partial C_{\mathrm{C}}}{\partial u} u_{\mathrm{ZP}},
$$

where $u_{\mathrm{ZP}}$ is the amplitude of the membrane's zero-point motion.

Using a simple circuit model (See Supplemental Material for details of the circuit simulation), we fit $\left|S_{21}\right|$ and extract $C_{\mathrm{C}} \approx 1.6 \mathrm{pF}$. Within the parallel plate capacitor approximation, $C_{\mathrm{C}} \sim \frac{\varepsilon_{0} a}{d}$, where $\varepsilon_{0}$ is the vacuum permit- 

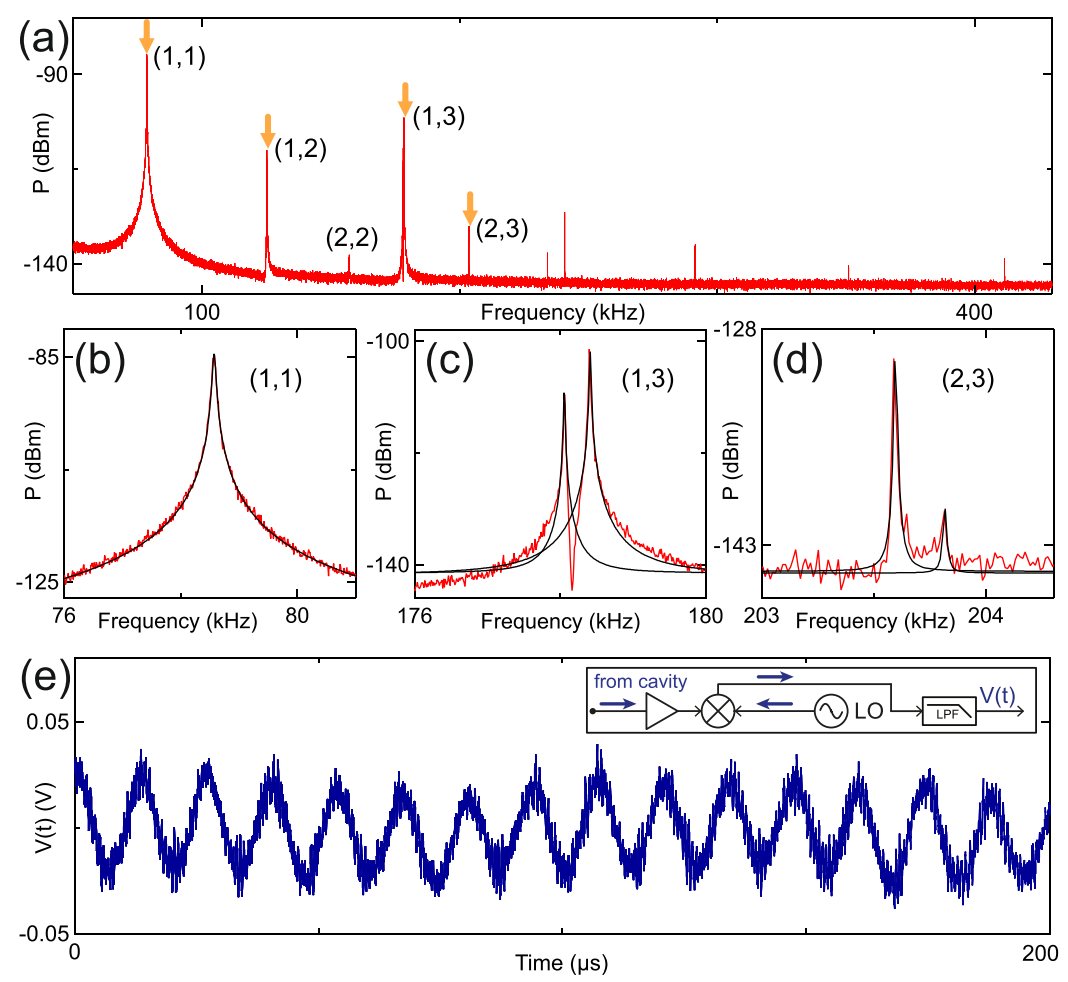

Figure 2. (a) Power spectrum as a function of frequency for the higher-frequency sidebands. A tone at $f_{\mathrm{C}}$ is applied with input power $P_{\mathrm{C}}=5 \mathrm{dBm}$ and white noise is injected via port 3 at $V_{\mathrm{M}}=2.7 \mathrm{~V}_{\mathrm{rms}}$ at electrode 1 . We observe several mechanical modes. The four mechanical modes visible in Fig. 1(d) are indicated with arrows. The mode at $\sim 157 \mathrm{kHz}$, labelled (2,2), is not visible in Fig. 1(d) due to a higher noise floor than in (a), which arises from a higher resolution bandwidth. Higher modes are not labelled because the deviation from theoretical ratios makes their identification ambiguous. (b-d) Zoom in showing the fundamental mode, and selected nearly degenerate modes. The black curves are Lorentzian fits. (e) Demodulated output signal as a function of time for the fundamental mode. The demodulation circuit is shown in the inset. (LO: local oscillator; LPF: low-pass filter). Input power is $P_{\mathrm{C}}=12 \mathrm{dBm}$ at $f_{\mathrm{C}}$ and the white noise is $V_{\mathrm{M}}=3.6 \mathrm{~V}_{\mathrm{rms}}$ at electrode 1 .

tivity, $a$ is the metallised area of the membrane and $d$ is the membrane-electrode gap. From this expression, we extract $d \sim 9 \mu \mathrm{m}$.

\section{Results}

To find the mechanical resonances, we drove the cavity on resonance $\left(f_{\mathrm{C}} \approx 209.2 \mathrm{MHz}\right)$ with input power $P_{\mathrm{C}}=5$ $\mathrm{dBm}$ at port 1 . Meanwhile, we excited the membrane's motion via port 3 , using a sinusoidal signal at frequency $f_{\mathrm{E}}$ and with amplitude $V_{\mathrm{M}}=3.6 \mathrm{~V}_{\mathrm{rms}}$ at electrode 1 . In order for the mechanical response to appear broader in the frequency spectrum, facilitating the detection of the mechanical modes, the excitation frequency at port 3 was modulated with a white noise pattern with a deviation of $200 \mathrm{~Hz}$. The power spectrum $P$ of the reflected signal at port 2 shows sidebands at $f_{\mathrm{C}} \pm f_{\mathrm{E}}$ due to non-linearities of the $\mathrm{rf}$ circuit giving rise to frequency mixing. The mechanical signal appears when $f_{\mathrm{E}}$ is close to a mechanical resonance, and is evident as a pronounced increase in sideband amplitude and width (Fig. 1(c)).

Figure $1(\mathrm{~d})$ shows the sideband at $f_{\mathrm{C}} \pm f_{\mathrm{E}}$ as a function of $f_{\mathrm{E}}$. The fundamental mode frequency $f_{1,1}$, which we will call $f_{0}$, is $\sim 77.9 \mathrm{kHz}$, giving an unresolved sideband ratio of $2 \pi f_{0} / \kappa \sim 3 \times 10^{-3}$, with $\kappa=2 \pi f_{\mathrm{C}} / Q_{\mathrm{E}}$ the cavity linewidth. As well as the fundamental mode, we observe less strong harmonics $f_{i, j}$ near the expected frequencies. The expected frequencies for higher harmonics theoretically satisfy the ratios $f_{i, j} / f_{0}=\frac{1}{\sqrt{2}} \sqrt{i^{2}+j^{2}}$ for integers $i$ and $j$ with symmetric roles as expected for a square membrane. Two of the sidebands are double peaked, evidencing nearly degenerate mechanical modes (insets Fig. 1(d)). The broken degeneracy could be due to imperfections in how the membrane was fabricated and fixed in place or uneven binding/deposition of the Al layer.

The entire set of mechanical resonances can be observed in a single measured power spectrum by driving the cavity at $f_{\mathrm{C}}$ and injecting white noise via port 3 . White noise excites the motion of the membrane at all frequencies, which is equivalent to raising the effective mechanical temperature. In this way, the root mean square (rms) displacement is increased, thereby facilitating the detection of mechanical modes. The noise signal has a bandwidth of $1 \mathrm{MHz}$, larger than the spectral width of the mechanical modes, and an amplitude $V_{\mathrm{M}}=2.7 \mathrm{~V}_{\text {rms }}$. Figure 2(a) shows the mechanical sidebands at $f_{\mathrm{C}}+f_{i, j}$. To distinguish mechanical sidebands from other parasitic signals, we increase $P_{\mathrm{C}}$ until we observe a frequency shift (See Supplemental Material for further details). The fundamental mode of the membrane is at $f_{0}=78.573 \pm 0.002 \mathrm{kHz}$. We fit each mechanical sideband with a Lorentzian (Fig. 2(b-d)). As in Fig. 1(d), we observe double peaked sidebands (Fig. 2(c,d)). 

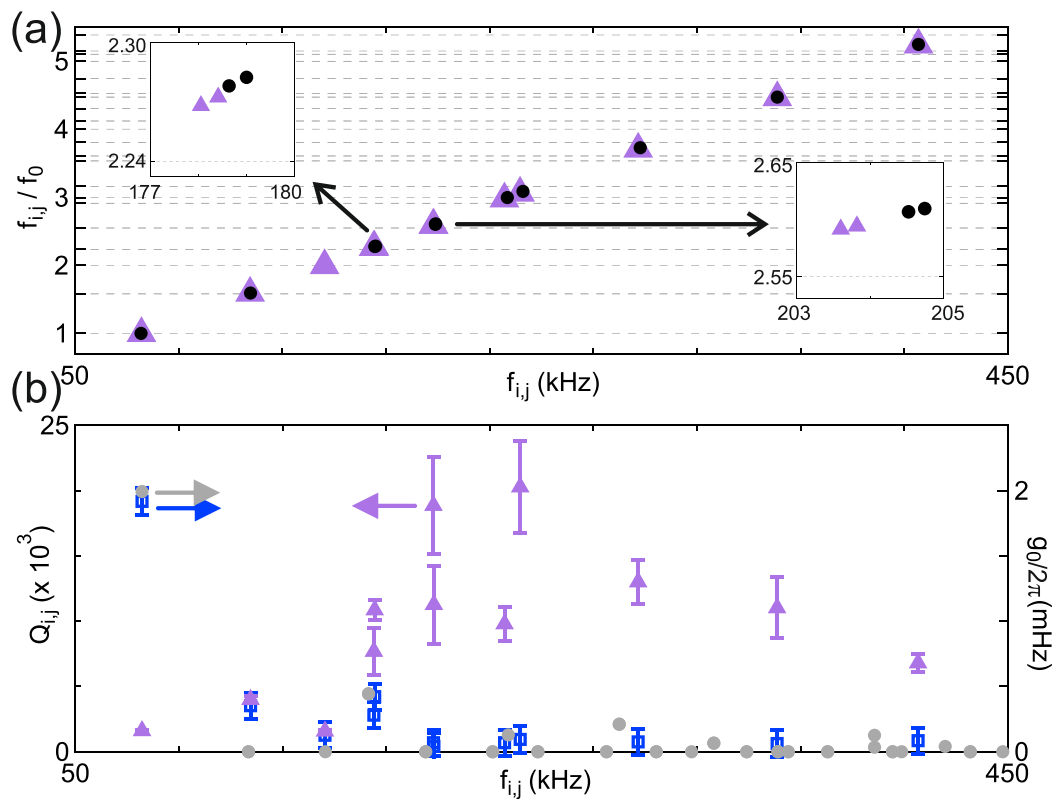

Figure 3. (a) Frequency ratios of the mechanical resonances extracted from Fig. 1(d) (circles) and Fig. 2(a) (triangles). Grid lines indicate theoretical ratios. Errors in frequency and frequency ratios are smaller than symbols. Insets: zoom-in showing selected nearly degenerate modes. (b) Triangles: Quality factors extracted from Lorentzian fits as in Fig. 2 for each mechanical mode. Error bars were obtained by combining fit results for different values of $V_{\mathrm{M}}$. Squares: Single-photon coupling strength calculated for each mechanical mode. Circles: Single-photon coupling strength estimated from a parallel capacitor model, corrected for different mode profiles, for $f_{i, j}$ obtained from theoretical ratios.

The broad cavity bandwidth allowed us to measure the actuated membrane's motion in real time. We excite the membrane with white noise whilst driving the cavity at $f_{\mathrm{C}}$. In order to record the motion in real time, the cavity output signal is mixed with a local oscillator. The output signal (Fig. 2(e)) shows clear sinusoidal oscillations evidencing the membrane's motion.

We plot $f_{i, j} / f_{0}$ as a function of $f_{i, j}$ in Fig. 3(a) for all mechanical resonances observed in Figs. 1(d) and 2(a). Lower frequency modes show better agreement with theoretical ratios than higher frequency modes. From the Lorentzian fits of each mechanical sideband, we extract the mechanical quality factors $Q_{\mathrm{i}, \mathrm{j}}$ and single photon coupling strengths $g_{0}$ (Fig. 3(b)). These values of $Q_{\mathrm{i}, \mathrm{j}}$, measured in the spectral response, are sensitive both to dissipation and dephasing ${ }^{20}$. The fundamental mode has a quality factor $Q_{0}=(1.6 \pm 0.1) \times 10^{3}$ and the highest quality factor measured was $(20 \pm 4) \times 10^{3}$ for the mode at $241 \mathrm{kHz}$. Different modes and even nearly degenerate mechanical modes have significant differences in their values of $Q_{i, j}$, as previously reported ${ }^{21,22}$. The values of $Q_{i, j}$ vary slightly as a function of $V_{M}$ ranging from 0.1 to $3.6 \mathrm{~V}_{\mathrm{rms}}$, but they do not show a specific trend (See Supplemental Material for data on the mechanical quality factors as a function of $\mathrm{V}_{\mathrm{M}}$ ). The error bars correspond to this deviation in the values of $Q_{i, j}$.

We extract $g_{0}$ for each mechanical mode from the effective thermomechanical power, i.e. from the area of the corresponding sideband in Fig. 3(b) (See Supplemental Material for details of the estimation of the single-photon coupling from noise measurements). For the first mechanical mode, we obtain $g_{0} / 2 \pi=1.9 \pm 0.1 \mathrm{mHz}$. The cavity drive was $5 \mathrm{dBm}$ at port 1 , yielding a multiphoton coupling strength $g / 2 \pi=\sqrt{n_{\mathrm{C}}} g_{0} / 2 \pi \sim 4.4 \mathrm{kHz}$, where $n_{\mathrm{C}}$ is the mean cavity photon number. As expected, the first mode couples more strongly than higher frequency modes, due to its mode profile and larger zero point motion (See Supplemental Material for details).

These values of $g_{0}$ can be compared with the predictions of Eq. 1 . Taking $C_{\mathrm{T}}$ and $d$ from the circuit model, and using that $\frac{\partial C_{\mathrm{C}}}{\partial u} \sim \frac{\varepsilon_{0} a}{d^{2}}$ for a parallel-plate capacitor (with a known prefactor to account for the mechanical mode profile of the membrane), gives a coupling strength $g_{0} / 2 \pi \approx 2 \mathrm{mHz}$ for the fundamental mode ${ }^{18}$. The estimated values of $g_{0}$ are similar to the ones extracted from the sideband powers.

We can estimate the vibrational amplitude sensitivity $S_{u}$ given an amplifier voltage sensitivity $S_{v}=\sqrt{k_{\mathrm{B}} T_{\mathrm{N}} Z_{0}}$, where $k_{\mathrm{B}}$ is the Boltzmann constant, $Z_{0} \equiv 50 \Omega$ and $T_{\mathrm{N}} \sim 293 \mathrm{~K}$ the system noise temperature. We can write $S_{u}=S_{v} /\left(\frac{\partial\left|S_{21}\right|}{\partial C_{\mathrm{T}}} \frac{\partial C_{\mathrm{C}}}{\partial u} V_{\text {in }}\right)$ where $V_{\text {in }}$ is the voltage at electrode 1 corresponding to $P_{\mathrm{C}}$. Taking $\frac{\partial\left|S_{21}\right|}{\partial C_{\mathrm{T}}}$ from the circuit model and $\frac{\partial C_{\mathrm{C}}}{\partial u} \sim 1.7 \times 10^{-7} \mathrm{~F} / \mathrm{m}$ extracted from the sideband's area corresponding to the fundamental mode, we obtain $S_{u}=0.4 \mathrm{pm} / \sqrt{\mathrm{Hz}}$. This value is comparable to that obtained for a suspended carbon nanotube device at cryogenic temperatures ${ }^{12}$.

Finally, we measure optomechanically induced transparency (OMIT) and absorption (OMIA), which are signatures of optomechanical coupling and demonstrate that the membrane's motion can be actuated by radiation-pressure alone. OMIT and OMIA are characterized by the emergence of a transparency or absorption window in $\left|S_{21}\right|$ when a strong drive tone $\left(f_{\mathrm{D}}\right)$ and a weak probe tone $\left(f_{\mathrm{P}}\right)$ are injected into the cavity, and 
(a)

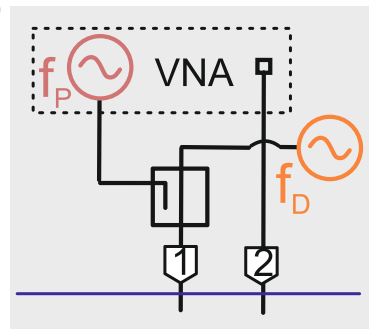

(c)

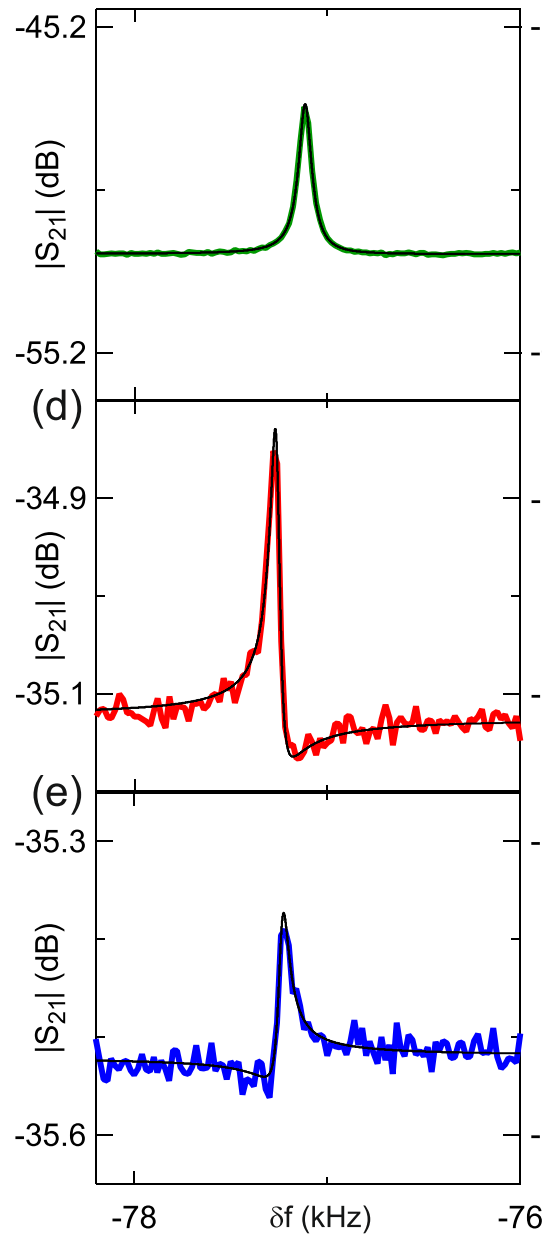

(b)
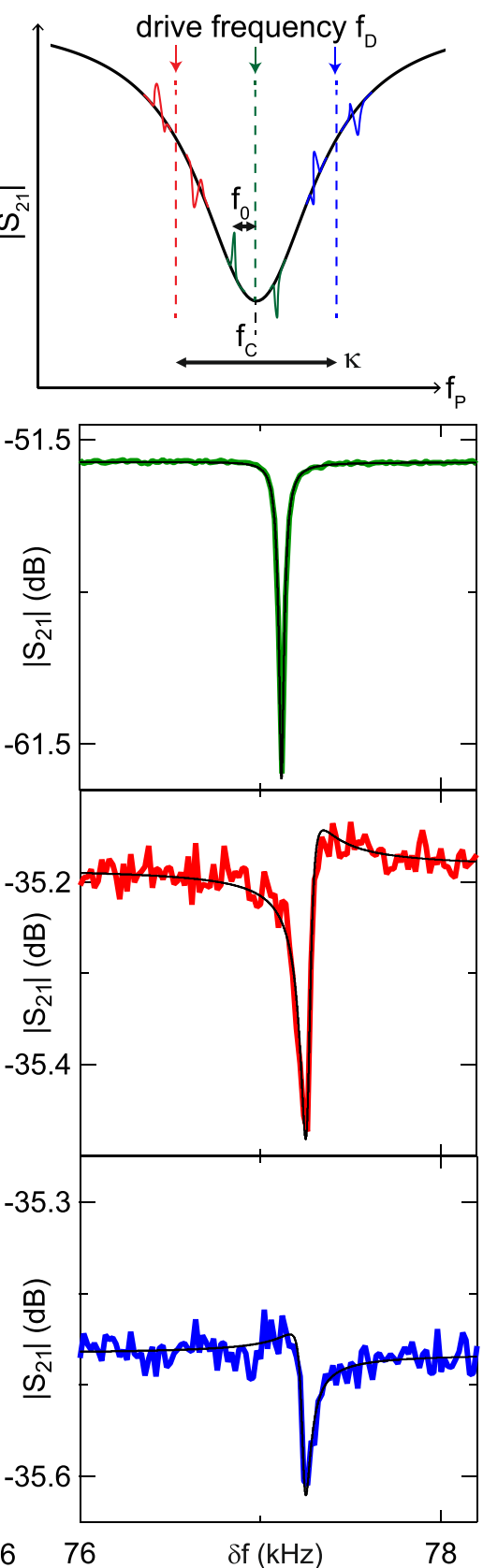

Figure 4. (a) Schematic of signal injection and detection for OMIT/OMIA measurements. A tone with frequency $f_{\mathrm{D}}$ and a tone with frequency $f_{\mathrm{P}}$ are injected through a directional coupler via port 1 . We monitor $\left|S_{21}\right|$ with a vector network analyzer. (b) Schematic showing each of the drive frequencies injected and a cartoon of the mechanical sidebands appearing at $f_{\mathrm{D}} \pm f_{0}$. Drive frequencies were $f_{\mathrm{D}} \sim f_{\mathrm{C}}$ (green), $f_{\mathrm{D}} \sim f_{\mathrm{C}}-\kappa / 2$ (red) and $f_{\mathrm{D}} \sim f_{\mathrm{C}}+\kappa / 2$ (blue). The corresponding mechanical sidebands are shown in panels (c-e) respectively. (c-e) Measured $\left|\mathrm{S}_{21}\right|$ as a function of $\delta f$ showing mechanical sidebands at $\delta f \sim-f_{0}$ (left panel) and $\delta f \sim f_{0}$ (right panel) for each of the values of $f_{\mathrm{D}}$ considered in (b). At port 1, the drive power was $5 \mathrm{dBm}$ and the probe power $-27.5 \mathrm{dBm}$. Black curves are a fit to the data.

the frequency difference between these tones $\delta f=f_{\mathrm{P}}-f_{\mathrm{D}}$ coincides with the frequency of a mechanical mode ${ }^{15}$. When this condition is met, the beat between the drive and the probe field excites the membrane's motion and the destructive (constructive) interference of excitation pathways for the intracavity probe field results in a transparency (absorption) window in $\left|S_{21}\right|$.

To show optomechanical control, we measured OMIT and OMIA by injecting a strong tone at frequency $f_{\mathrm{D}}$ and a weak tone at frequency $f_{\mathrm{P}}$ through a directional coupler at port 1 (Fig. $4(\mathrm{a})$ ). We injected three different drive frequencies (Fig. 4(b)); $f_{\mathrm{D}} \sim f_{\mathrm{C}}-\kappa / 2$ (red detuned), $f_{\mathrm{D}} \sim f_{\mathrm{C}}$ (resonant) and $f_{\mathrm{D}} \sim f_{\mathrm{C}}+\kappa / 2$ (blue detuned). When $f_{\mathrm{D}} \sim f_{\mathrm{C}}$, a peak (dip) is observed at $f_{\mathrm{D}}-(+) f_{0}$ (Fig. 4(c)). When $f_{\mathrm{D}} \sim f_{\mathrm{C}} \pm \kappa / 2$, we observe Fano-like features at $f_{\mathrm{D}} \pm f_{0}$ 
(Fig. 4(d,e)). We do not expect complete extinction or revival of the reflected signal as the system is well within the unresolved sideband limit.

We fitted OMIT and OMIA features by modelling the transmission of the probe field (See Supplemental Material for details of the estimation of the single-photon coupling from OMIT measurements). From the fit of the spectral features, we extract $f_{0}=77.2 \pm 0.1 \mathrm{kHz}, Q_{0}=(1.2 \pm 0.1) \times 10^{3}$ and $g_{0} / 2 \pi=2.3 \pm 0.3 \mathrm{mHz}$. Error bars were obtained by combining fit results of the six curves in Fig. 4(c-e). The values obtained for $f_{0}$ and $Q_{0}$ are similar to those extracted from the Lorentzian fits as in Fig. 2(b). The value of $g_{0}$ is in agreement with that extracted from Fig. 2(a) and estimated from the parallel plate capacitor approximation.

\section{Discussion}

To conclude, we have characterized several modes of a silicon nitride membrane with an off-resonant rf circuit at room temperature, deeper in the unresolved sideband regime than previously explored. Our cavity allows for the injection of noise to actuate the motion of the membrane and effectively increase its mechanical mode temperature. We achieve a sensitivity of $0.4 \mathrm{pm} / \sqrt{\mathrm{Hz}}$, a tenfold improvement to that reported in ref. ${ }^{14}$ although the smaller size of their mechanical resonator makes direct comparison difficult. Our results show that our on-chip platform can be used for membrane actuation and characterization. The readout circuit operates at a convenient frequency and does not require the cavity to be tuned into resonance with the membrane as in other approaches. It therefore has applications in mechanical sensing and microwave-to-optical conversion. Thanks to the large bandwidth of the cavity we have also measured the actuated membrane's motion in real time. Finally we have observed OMIT and OMIA, from which we obtained a separate measure of the frequency, quality factor and coupling strength of the fundamental mode.

\section{Data availability}

The data analysed during the current study are available from the corresponding author on reasonable request.

Received: 25 September 2019; Accepted: 13 January 2020;

Published online: 03 February 2020

\section{References}

1. Aspelmeyer, M., Kippenberg, T. J. \& Marquard, F. Cavity optomechanics. Rev. Mod. Phys. 86, 1391-1452, https://doi.org/10.1103/ RevModPhys.86.1391 (2014).

2. Purdy, T. P., Peterson, R. W. \& Regal, C. A. Observation of Radiation Pressure Shot Noise on a Macroscopic Object. Science 339, 801-804, https://doi.org/10.1126/science.1231692 (2013).

3. Purdy, T. P., Yu, P. L., Peterson, R. W., Kampel, N. S. \& Regal, C. A. Strong optomechanical squeezing of light. Phys. Rev. X 3, 031012, https://doi.org/10.1103/PhysRevX.3.031012 (2013).

4. Andrews, R. W. et al. Bidirectional and efficient conversion between microwave and optical light. Nat. Phys. 10, 321-326, https://doi. org/10.1038/nphys2911 (2014).

5. Bagci, T. et al. Optical detection of radio waves through a nanomechanical transducer. Nature 507, 81-85, https://doi.org/10.1038/ nature13029 (2014).

6. Moaddel Haghighi, I., Malossi, N., Natali, R., Di Giuseppe, G. \& Vitali, D. Sensitivity-Bandwidth Limit in a Multimode Optoelectromechanical Transducer. Phys. Rev. Appl. 9, 34031, https://doi.org/10.1103/PhysRevApplied.9.034031 (2018).

7. Yuan, M., Singh, V., Blanter, Y. M. \& Steele, G. A. Large cooperativity and microkelvin cooling with a three-dimensional optomechanical cavity. Nat. Commun. 6, 8491, https://doi.org/10.1038/ncomms9491 (2015).

8. Noguchi, A. et al. Ground state cooling of a quantum electromechanical system with a silicon nitride membrane in a $3 \mathrm{D}$ loop-gap cavity. New J. Phys. 18, 103036, https://doi.org/10.1088/1367-2630/18/10/103036 (2016).

9. Peterson, R. W. et al. Laser Cooling of a Micromechanical Membrane to the Quantum Backaction Limit. Phys. Rev. Lett. 116, 063601, https://doi.org/10.1103/PhysRevLett.116.063601 (2016).

10. Fink, J. M. et al. Quantum electromechanics on silicon nitride nanomembranes. Nat. Commun. 7, 1-10, https://doi.org/10.1038/ ncomms12396 (2016)

11. Lehnert, K. W. Introduction to Microwave Cavity Optomechanics BT - Cavity Optomechanics: Nano- and Micromechanical Resonators Interacting with Light. 233-252 (Springer Berlin Heidelberg, Berlin, Heidelberg, https://doi.org/10.1007/978-3-64255312-7_11(2014)

12. Ares, N. et al. Resonant Optomechanics with a Vibrating Carbon Nanotube and a Radio-Frequency Cavity. Phys. Rev. Lett. 117, 170801, https://doi.org/10.1103/PhysRevLett.117.170801 (2016).

13. Brown, K. R. et al. Passive cooling of a micromechanical oscillator with a resonant electric circuit. Phys. Rev. Lett. 99, 137205, https:// doi.org/10.1103/PhysRevLett.99.137205 (2007).

14. Faust, T., Krenn, P., Manus, S., Kotthaus, J. P. \& Weig, E. M. Microwave cavity-enhanced transduction for plug and play nanomechanics at room temperature. Nat. Commun. 3, 728, https://doi.org/10.1038/ncomms1723 (2012).

15. Weis, S. et al. Optomechanically induced transparency. Science 330, 1520-3, https://doi.org/10.1126/science.1195596 (2010).

16. Safavi-Naeini, A. H. et al. Electromagnetically induced transparency and slow light with optomechanics. Nature 472, 69-73, https:// doi.org/10.1038/nature09933 (2011).

17. Ojanen, T. \& Børkje, K. Ground-state cooling of mechanical motion in the unresolved sideband regime by use of optomechanically induced transparency. Phys. Rev. A 90, 013824, https://doi.org/10.1103/PhysRevA.90.013824 (2014).

18. Yong-Chun, L., Yun-Feng, X., Luan, X.-S. \& Wong, C. W. Optomechanically-induced-transparency cooling of massive mechanical resonators to the quantum ground state. Sci. China Phys. Mech. 58, 1-6, https://doi.org/10.1007/s11433-014-5635-6 (2015).

19. Ares, N. et al. Sensitive radio-frequency measurements of a quantum dot by tuning to perfect impedance matching. Phys. Rev. Appl. 5, 34011, https://doi.org/10.1103/PhysRevApplied.5.034011 (2016).

20. Schneider, B. H., Singh, V., Venstra, W. J., Meerwaldt, H. B. \& Steele, G. A. Observation of decoherence in a carbon nanotube mechanical resonator. Nat. Commun. 5, 5819, https://doi.org/10.1038/ncomms6819 (2014).

21. Zwickl, B. M. et al. High quality mechanical and optical properties of commercial silicon nitride membranes. Appl. Phys. Lett. 92, 103125, https://doi.org/10.1063/1.2884191 (2008).

22. Yuan, M., Cohen, M. A. \& Steele, G. A. Silicon nitride membrane resonators at millikelvin temperatures with quality factors exceeding $10^{8}$. Appl. Phys. Lett. 107, 263501, https://doi.org/10.1063/1.4938747 (2015). 


\section{Acknowledgements}

We acknowledge discussions with G. J. Milburn and support from EPSRC (EP/J015067/1), the Royal Society, the Royal Academy of Engineering and Templeton World Charity Foundation. The opinions expressed in this publication are those of the authors and do not necessarily reflect the views of Templeton World Charity Foundation. K.E.K. acknowledges travel funding from the Australian Research Council (CE110001013), and thanks the Oxford Materials Department for hospitality during the initial stages of this work.

\section{Author contributions}

A.N.P. and N.A. performed the experiment, analysed the data and prepared the manuscript with contributions from E.A.L. K.E.K. contributed to the theory, M.M. helped with device preparation. N.A. conceived the experiment. A.N.P., K.E.K., M.M., E.A.L., G.A.D.B. and N.A. discussed results and commented on the manuscript.

\section{Competing interests}

The authors declare no competing interests.

\section{Additional information}

Supplementary information is available for this paper at https://doi.org/10.1038/s41598-020-58554-x.

Correspondence and requests for materials should be addressed to N.A.

Reprints and permissions information is available at www.nature.com/reprints.

Publisher's note Springer Nature remains neutral with regard to jurisdictional claims in published maps and institutional affiliations.

(c) (i) Open Access This article is licensed under a Creative Commons Attribution 4.0 International License, which permits use, sharing, adaptation, distribution and reproduction in any medium or format, as long as you give appropriate credit to the original author(s) and the source, provide a link to the Creative Commons license, and indicate if changes were made. The images or other third party material in this article are included in the article's Creative Commons license, unless indicated otherwise in a credit line to the material. If material is not included in the article's Creative Commons license and your intended use is not permitted by statutory regulation or exceeds the permitted use, you will need to obtain permission directly from the copyright holder. To view a copy of this license, visit http://creativecommons.org/licenses/by/4.0/.

(c) The Author(s) 2020 